\title{
A Combined Latent Class and Trait Model for the Analysis and Visualization of Discrete Data
}

\author{
Ata Kabán and Mark Girolami, Member, IEEE
}

\begin{abstract}
We present a general framework for data analysis and visualization by means of topographic organization and clustering. Imposing distributional assumptions on the assumed underlying latent factors makes the proposed model suitable for both visualization and clustering. The system noise will be modeled in parametric form, as a member of the exponential family of distributions and this allows us to deal with different (continuous or discrete) types of observables in a unified framework. In this paper, we focus on discrete case formulations which, contrary to self organizing methods for continuous data, imply variants of Bregman divergencies as measures of dissimilarity between data and reference points and, also, define the matching nonlinear relation between latent and observable variables. Therefore, the trait variant of the model can be seen as a data-driven noisy nonlinear Independent Component Analysis, which is capable of revealing meaningful structure in the multivariate observable data and visualizing it in two dimensions. The class variant (which performs the clustering) of our model performs data-driven parametric mixture modeling. The combined (trait and class) model along with the associated estimation procedures allows us to interpret the visualization result, in the sense of a topographic ordering. One important application of this work is the discovery of underlying semantic structure in text-based documents. Experimental results on various subsets of the 20-News groups text corpus and binary coded digits data are given by way of demonstration.
\end{abstract}

Index Terms—Latent trait model, generative model, nonlinear mapping, topographic mapping, independent component analysis, clustering.

\section{INTRODUCTION}

$\mathrm{M}$ ETHODS of data analysis such as visualization, automatic classification, and structure discovery in large collections of data gain a growing practical importance in many domains. Generative models are frequently used as tools for discovering latent structure or latent causes in high dimensional observable data. Areas of applications include data mining, telecommunications, bioinformatics, fraud detection, information retrieval, and marketing analysis. In particular, the modeling algorithm introduced in this paper, is well-suited for unsupervised discovery of semantic structure from text databases.

Generative Topographic Mapping (GTM) [6] is a tool for data visualization and demonstrates the topographic organizing power of a generative model, assuming a specific non-Gaussian latent prior. However, by assuming a Gaussian noise model, it is suitable only for the analysis of continuous data. In many situations the observations are discrete or categorical. In [5], the idea of adapting the GTM for binary data was proposed. In [15], [16], the GTM model was reconsidered for binary data that is modeled as a multidimensional Bernoulli distribution. Empirical results demonstrated that the modified GTM is a valuable tool

- The authors are with the School of Information and Communications Technology, University of Paisley, Paisley, PA1 2BE, Scotland.

E-mail: \{ata.kaban, mark.girolami\}@wpmail.paisley.ac.uk.

Manuscript received 25 Apr. 2000; revised 2 Nov. 2000; accepted 2 Mar. 2001.

Recommended for acceptance by C. Brodley.

For information on obtaining reprints of this article, please send e-mail to: tpami@computer.org, and reference IEEECS Log Number 111997. for finding topical structure in binary coded text documents.

Related model descriptions of clustering [8], [7] and visualization models [43], [31], [32] can be found in the statistical literature. A latent variable model for discrete data analysis can be found in [22] and its topographical extension in [23], the idea of which is essentially a symmetrical decomposition of the data matrix.

In this paper, we construct the generative model in a general framework for outcomes from the exponential family of distributions for both the cases of discretized continuous non-Gaussian (latent trait) [13] ${ }^{1}$ and discrete categorical (latent class) latent priors. These distributional assumptions allow us to treat the clustering and visualization problem of different data types within the same formalism.

Adopting approximate Monte Carlo sampling from a fixed two-dimensional, uniformly distanced grid (as originally proposed in GTM) provides the same formalism for both trait and class model formulations. In the case of class modeling, the sampling is performed from unit vector coordinated corners of a hypercube and the algorithm learns independent prototype vectors that have categorical meaning without any ordering. From the latent trait

1. In the statistics literature, a continuous latent variable is a "trait" if it explains a categorical response. However, the usage of the term was extended in [39] for mixed (discrete and continuous) observed variables. As we are mainly focusing on modeling discrete and categorical response variables (observables), we use this term to distinguish continuous latent variables from categorical-even if the general form of our model may include variants suitable for continuous observable data. 
solution emerges a topographical ordering of the compressed images of the data points, by allowing a smooth nonlinear relation between observed and latent variables. This relation creates an appropriate non-Gaussian latent prior and at the same time implicitly defines a neighborhood function over the grid points, such that a principled parameter estimation method can be efficiently employed. The class model clustering provides probabilistic labels that are useful for interpreting the visualization result, in the sense of the topographic ordering provided by the trait solution.

Under a Gaussian noise assumption the proposed trait variant of the model would reduce to the standard GTM, while the class variant would reduce to Gaussian mixture modeling by Expectation Maximization (EM). However, we focus specifically on discrete data applications, the primary motivation of this work being to extend the trait model formulation as a tool for text-mining.

In Section 2, we present the generalized generative model. Section 3 presents the EM solution in the general case. Section 4 treats particular cases of various discrete distributions and shows the dissimilarity measures induced by the various noise models. Section 5 provides simulation results in hand written digit recognition and text mining, the final section summarizes the conclusions.

\section{General formulation of the Probabilistic Generative Model}

Let us denote by $\mathbf{D}=\left(d_{t n}\right)_{t=1 . . T, n=1 . . N}$ the observed data. The matrix $\mathbf{D}$ contains $N$ independent observations of the $T$-dimensional data vectors $\mathbf{d}$. The vector $\mathbf{d}_{n}$ will refer to one column of this matrix i.e., one observed data point. We make the standard assumption for compression-type generative models - that the observations are a noisy expansion of a hidden generative variable. Thus, we define the generation process as a mixture model. We will assume independence of observables conditioned on the latent variable, which has proved to be a reasonable simplifying assumption [35], expressing the aim that the latent generative factors should capture all the dependency which exists among the components of the observed data [45].

The distributional assumptions are known to have a great influence on the resultant technique. The probability distribution over the model noise affects the type of data the model is suited for whereas, the assumed latent prior determines the applicability of the model i.e., the statistical properties we are looking for when analysing the multivariate data. A generative model with non-Gaussian assumptions on the latent variables is also known as Independent Component Analysis (ICA) [21], [17], [19] different variants of which have been demonstrated to highlight potential meaningful structure in data.

\subsection{Modeling the Latent Variable}

The distributional assumption over the latent variable is in fact responsible for the type of the model and it must be set in accordance to the scope of the application. Some practical examples and the usefulness of a non-Gaussian latent prior, employed in this paper, are discussed below.
It is well-known for example, [44], [14] that assuming a Gaussian latent prior together with a Gaussian noise model results in the model known as Principal Component Analysis (PCA) which performs a dense coding of the observable data. From [45], [16], it is also evident that the Gaussian latent prior provides similar results, even if the noise model is not Gaussian. On the other extreme, assuming a maximally sparse discrete latent distribution, made up by the canonical basis vectors as possible vector values, results in an unconstrained mixture model (class model), appropriate for clustering data into disjoint classes.

Adopting non-Gaussian latent priors has been shown to reveal informative structure in the data [18], combining the advantages of the above two models. As an analytically specified continuous latent distribution would possibly result in intractable integrals, we adopt the sampling technique utilized in GTM [6], [5], [14], and will refer to this type of model as a latent trait model. The discretized formulation enables the trait model to be considered within the same formalism as the class model and thus allows for a principled topographical model formulation [6].

The sampling method proposed in [32], [31] for visualizing categorical variables, the "density network" was used for discovering correlations in protein sequences and the required topography was generated by assuming a particular latent density. In the GTM type formulation however, the latent densities and correlations between these are explicitly designed to stand for predefined homogeneous neighborhood relations on a $2 \mathrm{D}$ grid. This allows a more controlled visualization, set against a reference-grid of equidistant points.

We will briefly present both the latent class and latent trait models and we will use the former for providing the class labels for the visualization provided by the latter model.

\subsubsection{The Latent Class Model}

For clustering in mutually exclusive classes, the possible values of the $L$-dimensional latent variables $\mathbf{c}$ can be considered as a uniform sampling from the corners of a $K$-dimensional hypercube, which are columns of a $K$ dimensional identity matrix. These represent some nominal variables i.e., which take on class labels or topic labels. The latent dimension is thus $K$ and one (vector) value of $\mathbf{c}$ will be denoted by $\mathbf{c}_{k}$. Mathematically, the latent distribution is

$$
p(\mathbf{c})=\sum_{k=1}^{K} \delta\left(\mathbf{c}-\mathbf{c}_{k}\right) P\left(\mathbf{c}=\mathbf{c}_{k}\right)
$$

which is an unconstrained mixture model. The mixture components must satisfy the relation $\sum_{k=1}^{K} P\left(\mathbf{c}=\mathbf{c}_{k}\right)=1$, as they form a discrete distribution and will be considered as uniformly distributed if no other prior information is available. These can be later updated using the estimated posteriors.

\subsubsection{The Latent Trait Model}

Similar to GTM, the starting point for our latent variables will be a $2 \mathrm{D}$ latent $M \times K,(M=2)$ uniform grid of points, 
$\mathbf{X}$, mapped by a set of $L$ nonlinear and linear basis vectors $\Phi_{l}[6]$. Thus, in this case, the $l$ th component of the nonlinear image of the $k$ th latent grid point is $c_{l k}=\phi_{l}\left(\mathbf{x}_{k}\right)$, where $\mathbf{x}_{k}$ are columns of the matrix $\mathbf{X}$ or in equivalent matrix notation

$$
\mathbf{C}=\Phi(\mathbf{X})
$$

where $\mathbf{C}$ is an $L \times K$ dimensional matrix.

When utilizing nonlinear basis functions one has a free choice amongst smooth functions. Throughout this paper, we utilized the radial basis function set with constant unit variance, which gives a sparse positive sample distribution for $\mathbf{c}_{l}$, that is skewed to the origin. Sparse positive coding has also been shown in [28] to have the capacity of revealing underlying causes or factors in positive data and more specifically, in explaining the latent structure in the case of text document data [25].

It is interesting to note that as the latent trait algorithm will utilize the $\mathbf{c}_{k}$ vectors for reconstructing the observed data and the nonlinear mapping through the basis functions $\Phi$ is fixed a priori, a more intuitive interpretation is also possible besides that given in the original GTM model [6]: The nonlinear images $\mathbf{c}_{k}$ of the grid points can be considered as a sample approximation of some correlated, but non-Gaussian distributed continuous latent variables [14]. In this light, the role of the grid points is to provide equidistant arguments for the sampling from the image of $\Phi$. The distributions composed of these samples $c_{l k}$ are nonGaussian in general, and most importantly, this way of sampling induces a fixed correlation structure in the $L$-dimensional submanifold. This correlation structure is the one that provides a "neighborhood" relation [26] for topographical organization, as will be shown analytically in Section 3.

The discretized formulation is convenient from the perspective of computational tractability (integrals reduce to summations), as demonstrated in discrete linear model formulations within the Blind Source Separation (BSS) domain [3], [40]-for BSS with discrete sources, the $\mathbf{c}_{k}$ vectors are comprised of all possible combinations of the elements of the dictionary of the latent variable vector. In both cases, the latent variable $\mathbf{c}_{k}$ can take on a finite number $(K)$ of possible values and as such the same formal treatment is possible. Consequently, (1) is also the formal description of the latent distribution for the case of latent trait models. However, the latent trait model is a constrained mixture model [6].

\subsection{Distributional Assumptions Over the Noise Model}

The assumption over the distribution of the noise defines the type of data the model is suitable for and, also, implies the form of the link function required [34], which is linear only for the Gaussian case. Modeling the noise as independent and identically distributed (i.i.d.) Gaussian, provides an analytically tractable solution, which proved to be useful for modeling continuous data, but is clearly, inappropriate for discrete data.
For the sake of simplicity and generality, we will consider the exponential family of distributions for modeling the noise during the derivation of the algorithm. The Gaussian distribution is a special case that provides GTM for the latent trait model and a mixture of Gaussians (soft version of K-means clustering) [4] for the latent class formulation. We will emphasize particular cases that are suitable for categorical data, as these can also be modeled by members of the exponential family.

Thus, let $p\left(\mathbf{d}_{n} \mid \mathbf{c}_{k}\right)$ be from the exponential family of distributions. The exponential family of distributions has the functional form [1], [2], which depends on the cumulant function $G$

$$
p_{G}(\mathbf{x} \mid \theta)=\exp \{\theta \mathbf{x}-G(\theta)\} p_{0}(\mathbf{x}),
$$

where $\theta$ is called the natural (canonical) parameter of the exponential distribution, $G(\theta)=\ln \left(\int \exp (\theta \mathbf{x}) p_{0}(\mathbf{x}) d \mathbf{x}\right)$ is the cumulant function and is a strictly convex function of $\theta$ and $p_{0}(\mathbf{x})$ is a factor independent of the parameters. Products between vectors denote dot products.

In our conditional probability formulation of the noise $p_{G}\left(\mathbf{d}_{n} \mid \mathbf{c}_{k}, \mathbf{A}\right)$, the natural parameter will be assumed to be a parameterized linear mixing of the components of the possible latent vectors $\mathbf{c}_{k}$ thus, $\theta_{k}=\mathbf{A} \mathbf{c}_{k}$, where $\mathbf{A}$ is the parameter matrix of the trait model.

$$
p_{G}\left(\mathbf{d}_{n} \mid \mathbf{c}_{k}, \mathbf{A}\right)=\exp \left\{\mathbf{A} \mathbf{c}_{k} \mathbf{d}_{n}-G\left(\mathbf{A} \mathbf{c}_{k}\right)\right\} p_{0}\left(\mathbf{d}_{n}\right) .
$$

The gradient vector of the cumulant function with respect to the natural parameter $\theta_{k}=\mathbf{A} \mathbf{c}_{k}$, termed the expectation parameter, will be denoted by $\mathbf{m}_{k}$

$$
\mathbf{m}_{k}:=\mathbf{g}\left(\mathbf{A} \mathbf{c}_{k}\right)=\nabla_{\theta_{k}} G\left(\mathbf{A} \mathbf{c}_{k}\right),
$$

where $\nabla$. denotes the gradient operator and $\mathbf{g}($.$) is termed$ the link function [34].

By the first moment identity for log-likelihood functions applied to the exponential family of distributions [2], we know that $\mathbf{m}_{k}$ is the mean of class $k$ under the distribution $p\left(\mathbf{d}_{n} \mid \mathbf{c}_{k}\right)$.

$$
\mathbf{m}_{k}=E\left\{\mathbf{d} \mid \mathbf{c}_{k}\right\},
$$

where $\mathbf{d}$ denotes the random vector of observations and $E\{$.$\} is the expectation operator.$

We also know from the second moment identity for log-likelihood functions applied to the exponential family of distributions [2], that the expected value of the Hessian of the cumulant function with respect to $\mathbf{A c}_{k}$, is the covariance matrix of class $k$ under the distribution $p\left(\mathbf{d} \mid \mathbf{c}_{k}\right)$, i.e., the Fisher information matrix. This matrix will be denoted by $\mathbf{G}_{k}$.

$$
\mathbf{G}_{k}:=\nabla_{\theta_{k}} \mathbf{m}_{k}=\operatorname{Var}\left\{\mathbf{d} \mid \mathbf{c}_{k}\right\}
$$

\subsection{The State Space Form of the Model}

Summarizing, the model takes the following form:

$$
\mathbf{d}_{n}=\mathbf{g}\left(\mathbf{A} \mathbf{c}_{n}\right)+\mathbf{n},
$$

where $\mathbf{c}_{n}$ are the unobserved latent variables or sources, which take on $K$ possible values $\left\{\mathbf{c}_{k}\right\}_{k=1 . . K}$-i.e., 
non-Gaussianly distributed under the GTM-like latent trait assumption and a mixture of delta functions centered at corners of an $L$ dimensional hypercube under the latent class assumption. A can be considered as a loading matrix, and the mixture formed by the linear transform $\mathbf{A}$ is then nonlinearly transformed by $\mathbf{g}($.$) in the generative process, where \mathbf{g}($.$) , is defined in$ (5) and has its matching noise $\mathbf{n}$. If $\mathbf{n}$ is Gaussian, then $\mathbf{g}$ is the identity, and the GTM (which can also be viewed as a variant of noisy linear ICA [14]) is recovered. The latent trait model using non-Gaussian noise can be seen as a variant of nonlinear ICA with a data-driven nonlinearity, which contrary to the usual nonlinear ICA approaches, has its noise model aligned to the model's nonlinearity. As far as the latent class model is concerned, values of each $\mathbf{c}_{k}$ are canonical unit vectors and, thus, simply select one of the columns of matrix $\mathbf{A}$. Therefore, the $\mathbf{c}_{n}$ in (8) can be written outside the nonlinearity and the model becomes linear $\mathbf{d}_{n}=g(\mathbf{A}) \mathbf{c}_{n}+\mathbf{n}$. The model becomes in this case a parametric mixture model, where columns of $\mathbf{g}(\mathbf{A})$ are the class means. In this case, the algorithm for the model fitting is suitable for clustering.

Finally, it should be noted that a fully Bayesian treatment of the class modeling that includes the estimation of the number of classes is described in [8], [7]. This treatment does not have any topographical capability. The Bayesian framework could be extended to the constrained mixture case, for estimating the optimal number of grid points for the topographical mapping, however, this is outside the scope of this paper.

\section{THE EM SOLUTION}

As no ordering between the data points is assumed, the loglikelihood function of observables is given as

$$
l=\sum_{n=1}^{N} \log \left\{p\left(\mathbf{d}_{n}\right)\right\}=\sum_{n=1}^{N} \log \left\{\sum_{k=1}^{K} p\left(\mathbf{d}_{n} \mid \mathbf{c}_{k}\right) P\left(\mathbf{c}_{k}\right)\right\}
$$

where $\mathbf{c}_{k}$ are hidden variables (more precisely, the nonlinear images of the hidden grid point variables). Therefore, the maximization can be solved by the Expectation Maximization (EM) algorithm [11], an elegant and intuitive description of which can be found in [4], [38]. In accordance to the EM methodology, instead of maximizing the log likelihood we can maximize the relative likelihood [4], [11], [38], which does not contain the log of a sum. The relative likelihood between old and new parameters is the following:

$$
Q=\sum_{n=1}^{N} \sum_{k=1}^{K} p^{\text {old }}\left(\mathbf{c}_{k} \mid \mathbf{d}_{n}\right) \log \left\{p^{\text {new }}\left(\mathbf{d}_{n} \mid \mathbf{c}_{k}\right) P^{n e w}\left(\mathbf{c}_{k}\right)\right\},
$$

where

$$
r_{k n}:=p^{\text {old }}\left(\mathbf{c}_{k} \mid \mathbf{d}_{n}\right)=\frac{p\left(\mathbf{d}_{n} \mid \mathbf{c}_{k}\right) P\left(\mathbf{c}_{k}\right)}{\sum_{k^{\prime}=1}^{K} p\left(\mathbf{d}_{n} \mid \mathbf{c}_{k^{\prime}}\right) P\left(\mathbf{c}_{k^{\prime}}\right)}
$$

is estimated in the E step of the algorithm, being considered as constant in the maximization phase or M-step. It represents the responsibility of class $k$ in generating the observation $\mathbf{d}_{n}$, i.e., the probabilistic label of datum $\mathbf{d}_{n}$.

Replacing $p\left(\mathbf{d}_{n} \mid \mathbf{c}_{k}\right)$ by (4), (9) becomes:

$$
\begin{aligned}
Q=\sum_{n=1}^{N} \sum_{k=1}^{K} r_{k n}\left\{\mathbf{A} \mathbf{c}_{k} \mathbf{d}_{n}\right. & -G\left(\mathbf{A c}_{k}\right)+\log \left(p_{0}\left(\mathbf{d}_{n}\right)\right) \\
& \left.+\log \left(P^{n e w}\left(\mathbf{c}_{k}\right)\right)\right\}
\end{aligned}
$$

To complete the M-step in a latent trait model we need to maximize $Q$ in the latent trait parameter A. For clustering with a class model, we are mainly interested in determining $\mathbf{m}_{k}$ for all $k=1 . . K$. In this case, maximizing the likelihood in the expectation parameter gives a closed form result for the M-step. We will start our description by first presenting the latent trait solution.

\subsection{Latent Trait Solution}

In a latent trait model, the gradient of the distribution's cumulant function models the class expectations. Taking the derivative of $Q$ with respect to the parameter $\mathbf{A}$, we obtain from the element $A_{t l}$

$$
\frac{\partial Q}{\partial A_{t l}}=\sum_{n} \sum_{k} r_{k n}\left\{d_{t n}-g_{t}\left(\mathbf{A} \mathbf{c}_{k}\right)\right\} c_{l k},
$$

where $g_{t}$ is the $t$ th component of the vector argument vector function $\mathbf{g}($.$) , as was defined in (5). In equivalent matrix$ notation

$$
\frac{\partial Q}{\partial \mathbf{A}}=\left\{\mathbf{D} \mathbf{R}^{T}-\mathbf{g}(\mathbf{A C}) \mathbf{E}\right\} \mathbf{C}^{T},
$$

where $\mathbf{E}$ is a $K \times K$ diagonal matrix formed by $e_{k k}=\sum_{n} r_{k n}, \mathbf{R}=\left(r_{k n}\right)_{k=1: K ; n=1: N}$, and $\mathbf{D}$ is the previously defined data matrix. When the noise is isotropic Gaussian with unit variance then the matching function $\mathbf{g}($.$) is an$ identity, and, in this case, by setting the derivative equal to zero one gets the closed form M-step of GTM [6]:

$$
\mathbf{D R}^{T} \mathbf{C}^{T}=\mathbf{A C E C}^{T}
$$

In the general case, however, nonlinear optimization techniques may be required. This can be achieved, for example, using iterative least-square methods [34]. However, the Generalized EM (GEM) [38] algorithm is a more efficient choice, as convergence to a local maximum is guaranteed upon increasing and not necessarily maximizing the relative likelihood. From (13), we can obtain a simple gradient update for $\mathbf{A}$

$$
\Delta \mathbf{A} \propto\left\{\mathbf{D} \mathbf{R}^{T}-\mathbf{g}(\mathbf{A C}) \mathbf{E}\right\} \mathbf{C}^{T},
$$

which can be used in an inner loop in the M-step. Alternatively one can appeal to any nonlinear optimization techniques, some of which may require computation of the Hessian matrix, the elements of which are 


$$
\begin{aligned}
\frac{\partial^{2} Q}{\partial A_{t l} A_{t^{\prime} l^{\prime}}} & =\sum_{n} \sum_{k}-r_{k n} \frac{\partial}{\partial A_{t^{\prime} l^{\prime}}} g_{t}\left(\mathbf{A} \mathbf{c}_{k}\right) c_{l^{\prime} k} c_{l k} \\
& =\sum_{n} \sum_{k}-r_{k n}\left(G_{t t^{\prime}}\right)_{k} c_{l^{\prime} k} c_{l k},
\end{aligned}
$$

In the experiments reported in this paper, the gradient inner loop M-step was employed.

To observe that indeed the correlations between the $\mathbf{c}_{l}$ dimensions constitute the "neighborhood" [26] relations required for a topographical organization, consider how the natural parameter $\theta_{k}$ modifies under a gradient update of A. This is

$$
\theta_{k}^{\text {new }}=\mathbf{A}^{\text {new }} \mathbf{c}_{k}=\theta_{k}^{\text {old }}+\eta \sum_{n=1}^{N} \sum_{k^{\prime}=1}^{K}\left(\mathbf{d}_{n}-\mathbf{m}_{k^{\prime}}\right) r_{k^{\prime} n} \mathbf{c}_{k^{\prime}}^{T} \mathbf{c}_{k},
$$

which is analogous to the Self Organizing Map (SOM) [26] update. The width of the neighborhood relations made up by $\mathbf{C}^{T} \mathbf{C}$ is also controlled by the responsibility values $r_{k n}$, as discussed in [6].

The estimates of latent priors $P^{n e w}\left(\mathbf{c}_{k}\right)$ can be found by constrained maximization of $\mathrm{Q}$, as the probabilities must sum to one. Thus, we maximize $\tilde{Q}=Q+\lambda\left(\sum_{k=1}^{K} P^{\text {new }}\left(\mathbf{c}_{k}\right)-1\right)$, where $\lambda$ is the Lagrange multiplier. Taking the gradient with respect to $P^{\text {new }}\left(\mathbf{c}_{k}\right)$, setting it to zero and summing over $k$ leads to the standard update of the latent priors:

$$
P^{\text {new }}\left(\mathbf{c}_{k}\right)=\frac{1}{N} \sum_{n=1}^{N} r_{k n} .
$$

We now consider the latent class solution.

\subsection{Latent Class Solution}

A similar solution can be used for clustering by sampling from the corners of a hypercube rather than the grid. However, for class modeling, it is sufficient to maximize the likelihood with respect to the expectation parameter, which gives the following:

$$
\frac{\partial Q}{\partial \mathbf{m}_{k}}=\mathbf{G}_{k}^{-1} \sum_{n} r_{k n}\left(\mathbf{d}_{n}-\mathbf{m}_{k}\right),
$$

where $\mathbf{G}_{k}$ is the Fisher information matrix for class $k$, as was discussed in the previous section. Setting these equal to zero and solving for all $\mathbf{m}_{k}$ gives the Maximum Likelihood (ML) estimate for the expectation parameters, which in matrix format becomes

$$
\mathbf{M}=\mathbf{D} \mathbf{R}^{T} \mathbf{E}^{-1},
$$

where $\mathbf{R}$ is a $K \times N$ matrix formed by the responsibilities $r_{k n}, \mathbf{E}$ is a $K \times K$ diagonal matrix with the $k$ th diagonal element being $\sum_{n} r_{k n}$ and $\mathbf{M}$ is a $T \times K$ matrix having $\mathbf{m}_{k}$ as its $k$ th column. Equation (18) can also be expressed in terms of estimated second order sufficient statistics. Recognizing that $\mathbf{R}_{d c}:=\frac{1}{N} \mathbf{D} \mathbf{R}^{T} \approx E\left\{\mathbf{c d}^{T}\right\}$ and $\mathbf{R}_{c c}:=$ $\frac{1}{N} \mathbf{E} \approx E\left\{\mathbf{c c}^{T}\right\}$ are the respective sample covariance matrices (the latter holds for the class model, as $\mathbf{C}$ is an identity matrix), and $E\{$.$\} denotes expectation, (18) becomes$

$$
\mathbf{M}=\mathbf{R}_{d c} \mathbf{R}_{c c}^{-1} .
$$

The update of the latent priors is standard, as in the trait model's case.

\subsection{The Algorithm}

In conclusion, the outline of the combined latent trait and latent class modeling algorithm is given below. $\mathbf{R}_{\text {init }}$ will denote initial class-membership beliefs over the data set or a subset of it, and $\mathbf{D}_{\text {init }}$ will denote the (sub)set of data for which we have some initial beliefs or labels. If no prior information exists, then $\mathbf{D}_{\text {init }}=\mathbf{D}$ and $\mathbf{R}_{\text {init }}$ will be set randomly. Because a $2 \mathrm{D}$ projection from the trait model is sought for visualization purposes, the responsibilities of the vector values $\mathbf{c}_{k}=\Phi\left(\mathbf{x}_{k}\right)$ will be interpreted as being the posterior probabilities of the grid points $\mathbf{x}_{k}$.

\section{- Initialization.}

- for latent trait modeling:

$$
\mathbf{A}:=\text { random } ; \quad P\left(\mathbf{x}_{k}\right):=1 / K .
$$

- for latent class modeling:

$$
\mathbf{M}=\mathbf{D}_{\text {init }} \mathbf{R}_{\text {init }}^{T} \mathbf{E}_{\text {init }}^{-1} ; P\left(\mathbf{c}_{k}\right):=1 / K .
$$

\section{- Iterate until convergence.}

- $\quad$ E step. Estimate the responsibilities of the latent variables

$$
p^{\text {old }}\left(\mathbf{x}_{k} \mid \mathbf{d}_{n}\right)=\frac{p\left(\mathbf{d}_{n} \mid \mathbf{x}_{k}\right) P\left(\mathbf{x}_{k}\right)}{\sum_{k^{\prime}=1}^{K} p\left(\mathbf{d}_{n} \mid \mathbf{x}_{k^{\prime}}\right) P\left(\mathbf{x}_{k^{\prime}}\right)}=: r_{k n}^{\text {trait }}
$$

$$
p^{\text {old }}\left(\mathbf{c}_{k} \mid \mathbf{d}_{n}\right)=\frac{p\left(\mathbf{d}_{n} \mid \mathbf{c}_{k}\right) P\left(\mathbf{c}_{k}\right)}{\sum_{k^{\prime}=1}^{K} p\left(\mathbf{d}_{n} \mid \mathbf{c}_{k^{\prime}}\right) P\left(\mathbf{c}_{k^{\prime}}\right)}=: r_{k n}^{\text {class }}
$$

- $\quad$ M step.

- Parameter update for latent trait modeling:

- $\quad$ iterate until convergence

$$
\Delta \mathbf{A} \propto\left\{\mathbf{D} \mathbf{R}^{T}-\mathbf{g}(\mathbf{A C}) \mathbf{E}\right\} \mathbf{C}^{T},
$$

- or use any other nonlinear optimization procedure to increase the likelihood

- Parameter update for latent class modeling:

$$
\mathbf{M}=\mathbf{D} \mathbf{R}^{T} \mathbf{E}^{-1} .
$$

- Optionally, update the latent priors

$$
\begin{aligned}
& P^{\text {new }}\left(\mathbf{x}_{k}\right)=\frac{1}{N} \sum_{n=1}^{N} r_{k n}^{\text {trait }} \\
& P^{\text {new }}\left(\mathbf{c}_{k}\right)=\frac{1}{N} \sum_{n=1}^{N} r_{k n}^{\text {class }} .
\end{aligned}
$$

The initialization can be random or data-dependent (class membership beliefs obtained as outputs from another classifier) [35]. A useful alternative, in the case when there are a (small) number of human labeled instances available, 
the initialization step can make use of these for initializing the EM iterations from a closer (to the maximum) starting point, i.e., one can build a supervised method in the initialization stage [37], [24]. This is particularly useful when the observable data are high dimensional and sparse. In this case, the dependency of the EM algorithm on initializations is recognized as being even more pronounced [42].

Note that the algorithm is of the same form for all data types, i.e., for all noise distributions from the exponential family. However, care has to be taken in the case of sparse data, because sparsity poses certain statistical problems and consequently the ML estimation of the expectation parameter requires further corrections (see the following section). What varies with different data types is the nonlinear function $\mathbf{g}($.$) in the latent trait M-step. This is$ strictly related to the data point's likelihood in computing the E step, i.e., to the matching noise, or in other words, to the dissimilarity measure between a datum and reference point, adopted by the particular model. This dissimilarity, in both SOM [26] and GTM is the Euclidian distance-in SOM by being nonprobabilistic and in GTM by dealing with continuous data and, therefore, assuming Gaussian noise. In this paper, however, by focusing specifically on discrete members of the exponential family, the dissimilarity measures become variants of Bregman divergences [1], [2]. These divergence measures together with the appropriate noise assumptions in the case of discrete data modeling will be briefly reviewed in the following section. They result directly from the model instantiations and are implicitly defined by the probabilistic assumptions over the model noise.

\subsection{Implication of Data Sparseness in Generative Class Modeling}

As the class model is linear (as pointed out in Section 1), in the case where there is a one to one correspondence between mixture components and class labels, the model is capable of clustering only linearly separable classes. Sparse data has this property, revealed, for example, in the text classification domain by the success of linear classifiers on textual data [12], [49]. For binary coded data, this restriction can be more pronounced. To give an illustration, we recall that binary data signifies that each observable vector is a corner of a $T$-dimensional unit cube and that the number of linearly separable partitions of a $\mathrm{T}$ dimensional cube is $\mathcal{O}\left(2^{T^{2}}\right)$, while the number of total partitions is $2^{2^{T}}$ [41]. So the number of linearly separable partitions decreases rapidly with increasing dimensionality. Therefore, high-dimensional binary data must be very sparse to be linearly separable. This is probably the reason why Bernoulli class modeling was found consistently [33], [46] inferior to multinomial class modeling for high dimensional data. Note that multinomial class modeling makes use of the available frequency information.

While this linear separability is a welcome feature of sparse high-dimensional data, the sparsity poses statistical problems and so requires further corrections to the Maximum Likelihood solution. The problem is the so-called "zero counts problem," also encountered in Naïve Bayes (NB) classifiers, which means that there exist components of the outcomes that never occur in some classes. Therefore, for these nonoccurring components the corresponding component of the classes' expectation parameter will be zero, cancelling out the instance's class-probability, because it is modeled as factorable.

There are two main solutions to this problem in NB classifier practice, each having a number of variants. An extended empirical comparative review can be found in [27]. The first idea is to replace the zero count with a small number. The second, statistically founded solution [20] is Laplace correction or Laplace smoothing, which comes from imposing a conjugate prior on the class model's parameters. In practice, for a $k$-valued problem this consists of making the uniform prior assumption over all $k$ possible values. Formally, this means the following substitution:

$$
\frac{n}{N} \longrightarrow \frac{n+f}{N+k f}
$$

where $k$ is the number of possible values in a component (e.g., $k=2$ for binary data). All that is needed then, for applying Laplacian smoothing, is to write the expectation parameter in terms of sample probabilities in the case of the concrete noise distributions. $N$ is the number of instances, $n$ is the number of matches and $f$ is a predefined number. In [27], it is empirically shown that the NB's accuracy is highly dependent on the choice of $f$. While a common choice is $f=1$, leading to the interpretation that we are adding $k$ extra draws, each having probability of $1 / k$, in [27] it was found that $f=1 / N$ yields superior results.

We note that latent trait modeling naturally overcomes the zero count problem by modeling the expectation parameter as a nonlinear function that asymptotically reaches the value of zero.

We have theoretically described a generalized probabilistic modeling framework for unsupervised visualization and clustering of multivariate data. The general model presented provides a formal and unified method of model description and parameter estimation. It was noted that one such particular case of our model, resulting by choosing a Gaussian noise model, yields the original GTM algorithm [6]. The remainder of the paper will consider the modeling of discrete multivariate data in particular.

\section{Particular Cases in Modeling Discrete DATA}

In this section, we concentrate on those particular cases of the presented general model, where the distributional assumptions for $p\left(\mathbf{d}_{n} \mid \mathbf{c}_{k}\right)$ are suitable for representations of categorical and discrete data. For more complex applications with mixed types of data [43], the appropriate likelihood terms should simply be multiplied, due to the conditional independence assumption given the latent variable.

This section concerns both trait and class models. Besides selecting a particular cumulant function $G$ for (4) to obtain the individual distributions on the noise, we also point out the dissimilarity measures between data and reference vectors implied by each distributional assumption. For all the discrete data cases, these measures are variants of Bregman divergences [1]. Note that, each particular class 
model performs parametric mixture modeling of a certain mixture distribution and can be viewed as soft versions of K-means clustering, where the "distance" used in assigning instances to latent entities is directly generated from the assumed probabilistic model. The same is true for the latent trait modeling algorithm, with the essential difference that the latter performs a constrained parametric mixture modeling, because the centers cannot move separately but only through the parameter of the nonlinear mapping from a latent grid space to data space [6]. Thus, unlike the original GTM, the constrained mixtures are now non-Gaussian, and as a direct consequence, the distances between data and reference points are not Euclidean, but are instead, Bregman divergences which also directly arise from the probabilistic assumption over the model noise. In the Appendix, we have given the technical details concerning the use of discrete members of the exponential family of distributions within the presented framework.

\subsection{Text Data Modeling}

There are two event models in the generative representation of text documents [33]. The multivariate Bernoulli event model represents each document by a vector of binary components, each component indicates whether or not a certain term occurs in that document. The statistical event is the document. The multinomial event model, in turn, represents each document as a set of word events, as $\left|\mathbf{d}_{n}\right|$ independent trials from a "bag of words," where $\left|\mathbf{d}_{n}\right|$ is the length of the document. The order of the words is lost, however, the number of occurrences is captured. In this case, each document is, in fact, coded as a histogram of word occurrences. This representation has been found superior to the Bernoulli in both classification [33] and clustering [46], [47]. Normalizing the document length has the benefit of preventing longer text documents capturing more importance than the shorter ones. In other words, an occurrence of a word in a short document should obviously be treated as more important than an occurrence of a word in a long text. We therefore, will investigate the multinomial noise based latent trait model as an extension to the Bernoulli formulation [16].

The Poisson distribution can also be used in modeling count data. However, in the specific case of text based information retrieval it has been proposed and found to be inefficient [29], [36], [9] on term frequency count data, because of the mismatch between possibly long tailed word distributions over documents and the Poisson distribution [36]. However, the two-valued Poisson mixture model was utilized as a sparse substitute of the Bernoulli mixture model for clustering and was found to be less sensitive to initializations in the case of sparse data [24].

\section{EXPERIMENTAL Results}

Simulations were carried out on binary handwritten digit data $^{2}$ and various subsets of the 20-Newsgroups text corpus, ${ }^{3}$ in both binary and word count-based representations. The 20-Newsgroups text base consists of 20,000 docu-

2. http://www.ics.uci.edu/mlearn/MLSummary.html.

3. http://www.cs.cmu.edu/textlearning/. ments from usenet communications on various topics, arranged in 20 classes, and is diverse enough to enable various settings for testing the algorithms. The experiments are designed to compare and find the most appropriate probabilistic formulation for given data and to investigate the potential power of the trait model in finding meaningful structure in the multivariate data. Specifically, we were interested in finding semantic structure in textual data, by means of building up a two-dimensional semantic map on which we can visualize the text document instances in a topographic ordering.

\subsection{Documents Data Mining}

The most important questions from the perspective of this work concern

1. The general abilities of the trait models in revealing some underlying structure in multivariate data.

2. Whether or not the proposed multinomial trait model brings any improvement over the Bernoulli by allowing us to incorporate term frequency, or eventually weighted term frequency information in the case of the latent trait model.

3. To see whether they can indeed find meaningful semantic organizations, word groupings and topographical visualization.

In all experiments, the model parameters were set as follows: We chose a $10 \times 10$ uniform grid mapped onto 25 Gaussian basis functions having unit variance.

For illustrating the algorithms in the first experiment, we have chosen four document classes on specifically distinct topics: "sci.crypt," "sci.space," "sci.med," and "soc.religion.christian." Arbitrarily, 100 documents were used from each of these classes and a dictionary size of 100 terms was utilized. We mention that the relevant term selection procedure is another extensive area of research [48], [30], [42], which is outside of the scope of this paper. Because the aim of this experiment was mainly evaluating the possible models, the terms making up the documents features were chosen for this experiment by the Mutual Information (MI) [10] with human class labels. This term selection method is, however, possible in practice only if there are labeled instances available. In Fig.1 we can see the posterior means obtained on the training set (i.e., the $2 \mathrm{D}$ points of the matrix $\mathbf{R}^{T} \mathbf{X}$, where $\mathbf{R}$ is the $K \times N$ responsibility matrix and $\mathbf{X}$ is the $K \times 2$ matrix containing the grid-points) given by the individual trait models on the same data. The different class labels are shown with different symbols on the plots. We can see that each model has found a valid meaningful mapping, as related document instances are clumped together on the maps, while those concerning different topics are mapped further from each other. The original GTM was also run on this data for comparison. Indeed, it can be seen that the theoretical advantages of versions of the model proposed in this paper are reflected in the visually discriminative quality of the mappings.

Fig. 2 shows the posterior means on new, unseen test data using the previous parameterized models. Again 100 documents were presented from each class. The 

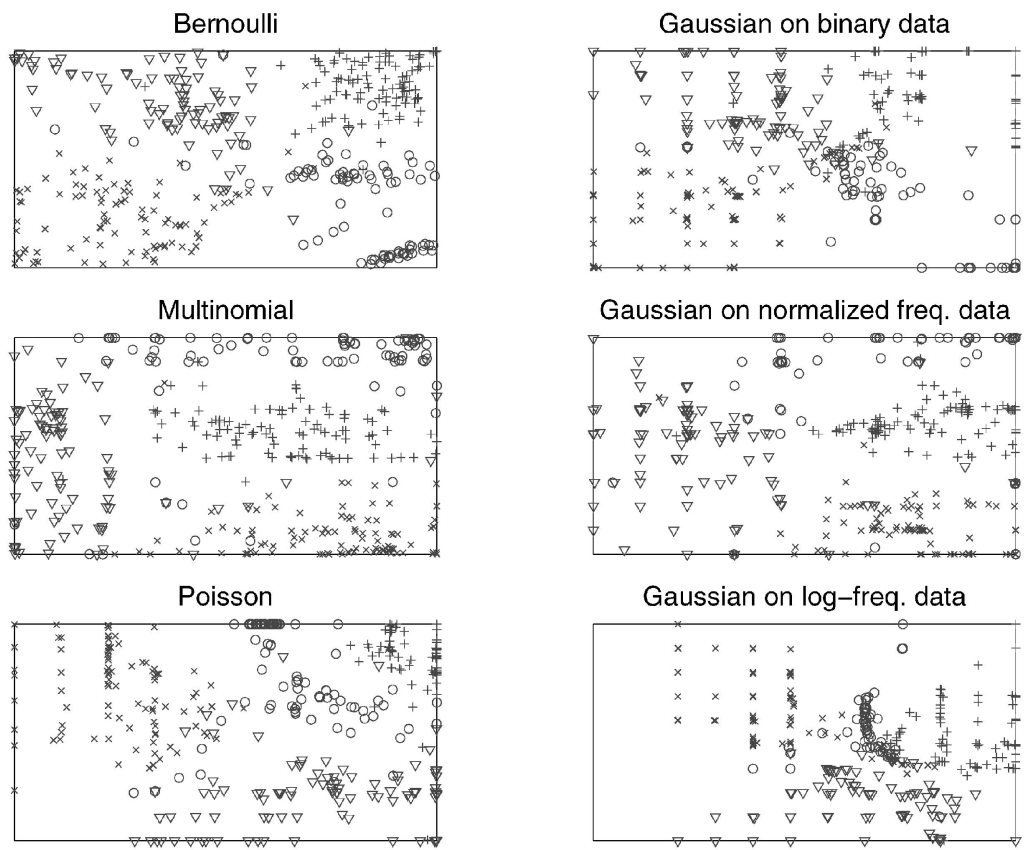

Gaussian on normalized freq. data

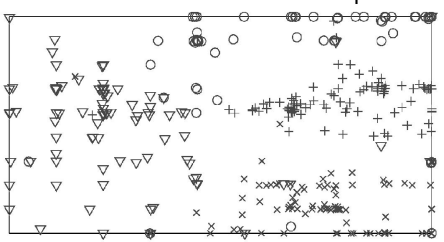

Gaussian on log-freq. data

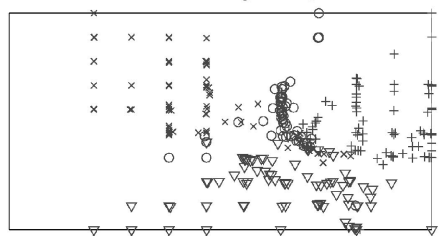

Fig. 1. Posterior means provided by the different probabilistic latent trait algorithms on training data using four distinct document classes from the 20-Newsgroups corpus: $x=$ "sci.crypt," $0=$ "sci.med," $\nabla=$ "sci.space," and + = "soc.religion.christian." The right plots show the corresponding results if using the theoretically inappropriate, but practically more simple original Gaussian GTM algorithm. The same latent settings and parameter initializations were used for each model.
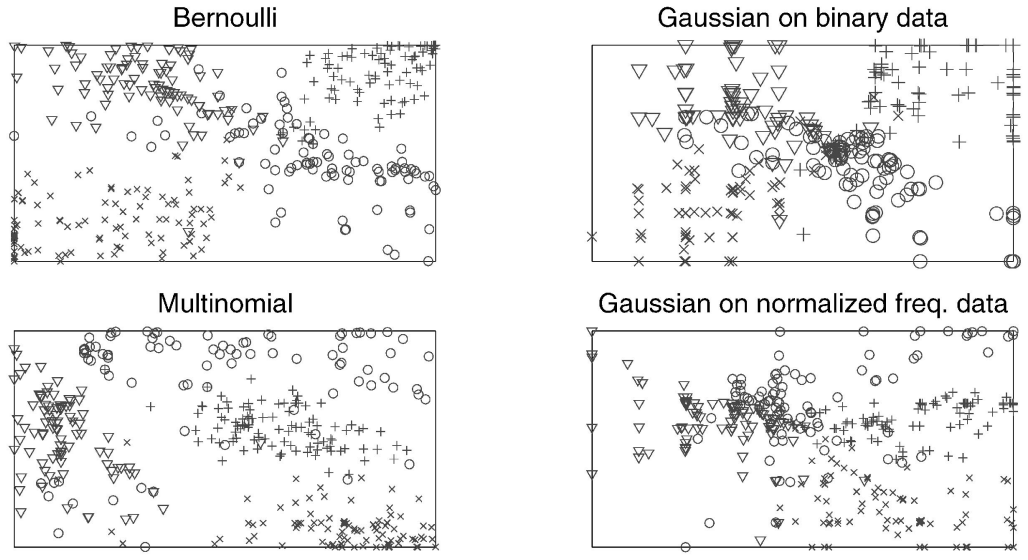

Gaussian on normalized freq. data
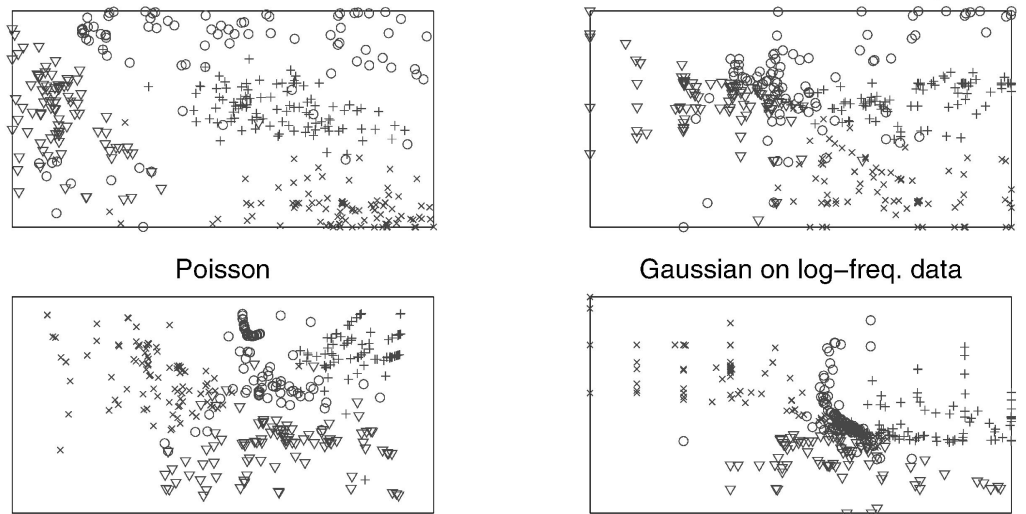

Gaussian on log-freq. data

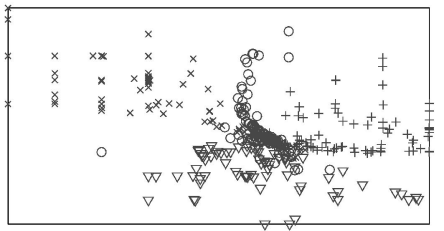

Fig. 2. Posterior means provided by the different probabilistic latent trait algorithms on test data using four distinct document classes from the 20-Newsgroups corpus: $\times=$ "sci.crypt," rmo = "sci.med," $\nabla=$ "sci.space," and $+=$ "soc.religion.christian." The same latent settings and parameter initializations were used for each model.

superiority of the theoretically appropriate model formulations is also reflected in the "generalization" capability. Moreover, the multinomial model empirically proves to be qualitatively superior for text modeling in these experiments-this can be evaluated visually on both Fig. 1 and Fig. 2-i.e., the mapped corpus has organized in more separated regions or classes. The observation of the multinomial model yielding superior results on text data was also reported in the literature for both supervised and unsupervised text-classification [37], [46].

The algorithms were also tested using an unsupervised term selection method, based on Zipf's law [42], i.e., retaining in the dictionary only the terms that occur more than a certain number of times, after removing the "stopwords" (words like "the," "is," etc.). Different dictionary sizes were investigated for completeness, however, it is a known fact that mixture modeling becomes less efficient as 

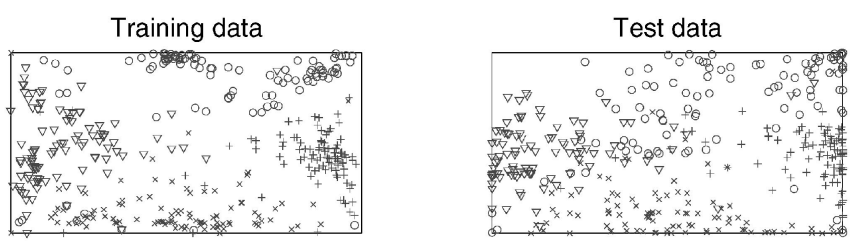

Fig. 3. Training and test data results with the multinomial model on text data using higher dimensional dictionary $(6,067$ terms).

the dimension of the dictionary increases [42]. However, we found the multinomial model more tolerant to both the suboptimal term selection and the curse of dimensionality. This finding is consistent with reported comparative classification and clustering results in the literature [33], [46], [42], [47]. Fig. 3 shows the best results (chosen visually from a series of Monte Carlo runs), on both training and testing sets, obtained with the multinomial model on the same data and using a dictionary size of 6,067 terms, chosen only by frequency of occurrence.

For the final experiment with text data the frequency count information gives more discrimination between otherwise possibly overlapping classes. In this case, the trait version with a count-based noise model formulation may provide superior results to that which considers only binary occurrence information. We chose three classes, such that two of them were strongly overlapping: "comp.sys.ibm.pc.hardware," "comp.sys.mac.hardware," and "sci.med." We arbitrarily took 200 documents from each class and a dictionary size of 150 terms was used. The dictionary was again advantageously chosen by MI, therefore, this dictionary size should be sufficient [42] to capture the structure of the corpus. Fig. 4 shows comparatively the 2D mapping obtained by the Bernoulli and multinomial trait model variants. Inspecting the lists of the most probable words, associated to the different grid points-i.e., the top of the reference vectors $\mathbf{g}\left(\mathbf{A} \mathbf{c}_{k}\right)$ which have been formed-we can recognize meaningful word groupings. Some of these lists are shown on Fig. 4. We can also observe that grid points that lie in one of the classes central region store meaningful keywords in their lists of the most probable words whereas, those lying in an overlapping area store words that occur in documents concerning the junction of these discussion topics in both models. For example, documents regarding the negative effects of computers on school children and those concerning the use of computers in medicine were mapped at the junction between the areas concerning computers and medicine. The above comments were made after exhaustive inspection of the reference vectors which have been estimated by the algorithm for evaluation purposes, thus demonstrating that the presented model can indeed consistently reveal the semantic structure of the corpus on a 2D map. The tables of the exhaustive reference-vectors can be seen on Fig. 5 and Fig. 6, respectively. These $10 \times 10$ tables show the top lists of the most probable words represented by all the $10 \times 10$ grid points in these two experiments. Each cell of the table corresponds to one grid point. For the ease of following for the reader, we traced some orientative boundaries between regions which treat different topics. We can indeed follow a distinct topographic ordering of the reference-vectors: Neighboring grid points have many words in common and words grouped together arise from the same subject
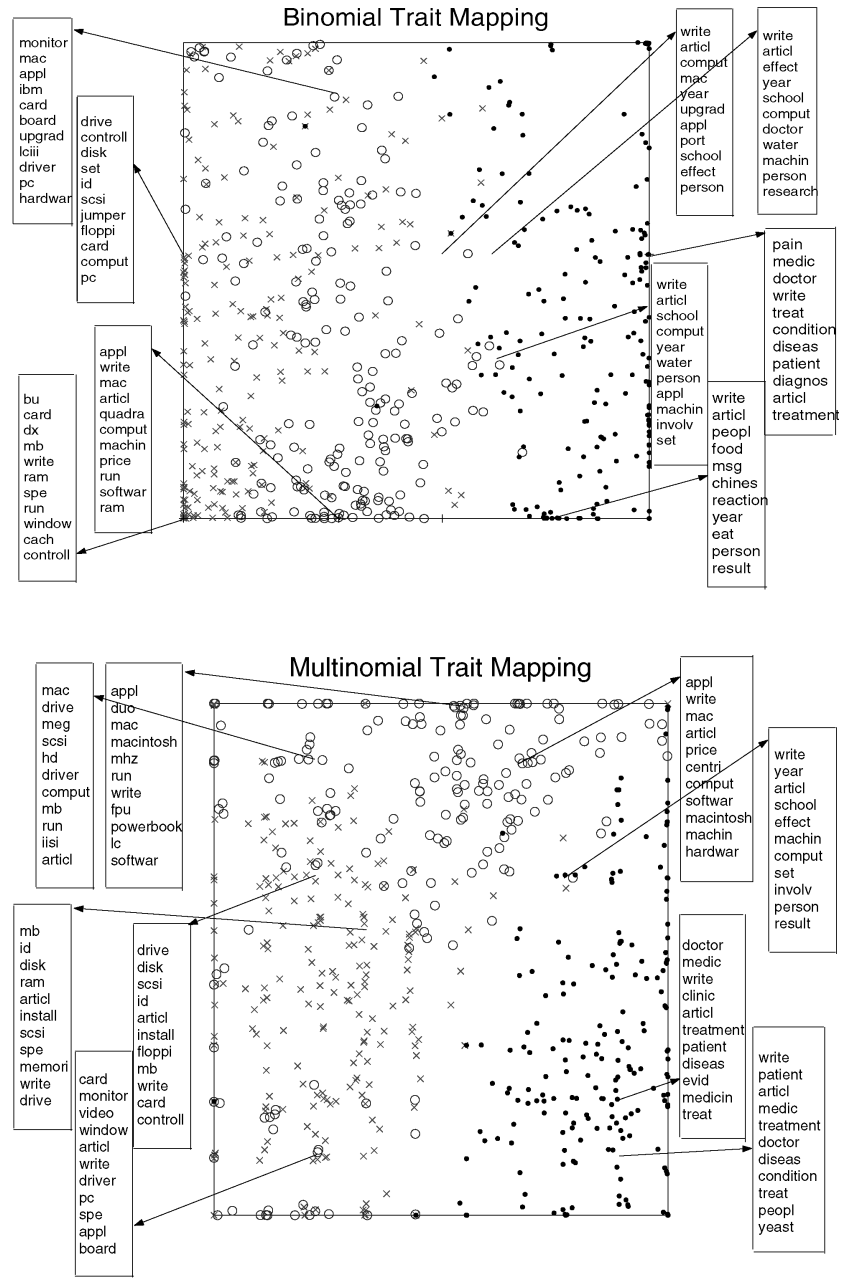

Fig. 4. Comparative posterior means provided by the Bernoulli and multinomial latent trait algorithm on two overlapping ( $\times=$ "comp.sys.ibm.pc.hardware," rmo = "comp.sys.mac.hardware") and one distinct (.= "sci.med") classes and inspecting some of the grid points for the most probable words.

area. However, in the differentiation of the two overlapping classes, the multinomial model's result is clearly superior. This can also be seen from the mapping of documents (Fig. 4). The Bernoulli trait model clearly distinguishes between computer science related and medicine related regions. It contains some regions inside the computer's subject that are specifically related to PCs and to Macintosh, respectively, but in most of the region PC and Macintosh related subjects coexist. In turn, the multinomial mapping has separated more distinctly even the somewhat overlapping computer-related topics by mapping them to distinct regions. We can follow on Fig. 6 in the upper region of the grid keywords regarding Macintosh hardware, in the left region keywords concerning PC hardware and the rightmost area is concerned with the PC. Note that the boundaries we traced are just approximative and are not unequivocal but the passing from a region to another is rather gradual.

Inspecting further Fig. 6, we can distinguish subpartitioning within one newsgroup's area talking about related but slightly distinct subjects, such as common health in the upper part of the medicines range whereas, more specific medical terms come in the right lower corner. Inside PC hardware 


\begin{tabular}{|c|c|c|c|c|c|c|c|c|c|}
\hline $\begin{array}{l}\text { card } \\
\text { board } \\
\text { slot } \\
\text { video } \\
\text { isa } \\
\text { Ic }\end{array}$ & $\begin{array}{l}\text { card } \\
\text { board } \\
\text { video } \\
\text { monitor } \\
\text { slot } \\
\text { appl }\end{array}$ & $\begin{array}{l}\text { monitor } \\
\text { video } \\
\text { card } \\
\text { board } \\
\text { appl } \\
\text { window }\end{array}$ & $\begin{array}{l}\text { monitor } \\
\text { video } \\
\text { card } \\
\text { appl } \\
\mathrm{ibm} \\
\text { window }\end{array}$ & $\begin{array}{l}\text { monitor } \\
\text { research } \\
\text { ibm } \\
\text { video } \\
\text { card } \\
\text { result }\end{array}$ & $\begin{array}{l}\text { research } \\
\text { monitor } \\
\text { risk } \\
\text { result } \\
\text { ibm } \\
\text { oral }\end{array}$ & $\begin{array}{l}\text { research } \\
\text { write } \\
\text { articl } \\
\text { oral } \\
\text { sci } \\
\text { drug }\end{array}$ & $\begin{array}{l}\text { write } \\
\text { articl } \\
\text { gordon } \\
\text { bank } \\
\text { geb } \\
\text { pitt }\end{array}$ & $\begin{array}{l}\text { gordon } \\
\text { bank } \\
\text { geb } \\
\text { pitt } \\
\text { write } \\
\text { articl }\end{array}$ & $\begin{array}{l}\text { gordon } \\
\text { bank } \\
\text { pitt } \\
\text { geb } \\
\text { articl } \\
\text { write }\end{array}$ \\
\hline $\begin{array}{l}\text { card } \\
\text { board } \\
\text { slot } \\
\text { comput } \\
\text { port } \\
\text { pc }\end{array}$ & $\begin{array}{l}\text { card } \\
\text { board } \\
\text { mac } \\
\text { pc } \\
\text { hardwar } \\
\text { comput }\end{array}$ & $\begin{array}{l}\text { monitor } \\
\text { mac } \\
\text { board } \\
\text { card } \\
\text { appl } \\
\text { pc }\end{array}$ & $\begin{array}{l}\text { monitor } \\
\text { mac } \\
\text { appl } \\
\text { ibm } \\
\text { card } \\
\text { board }\end{array}$ & $\begin{array}{l}\text { monitor } \\
\text { research } \\
\text { mac } \\
\text { ibm } \\
\text { upgrad } \\
\text { appl }\end{array}$ & $\begin{array}{l}\text { research } \\
\text { monitor } \\
\text { result } \\
\text { risk } \\
\text { drug } \\
\text { ibm }\end{array}$ & $\begin{array}{l}\text { research } \\
\text { write } \\
\text { drug } \\
\text { articl } \\
\text { result } \\
\text { effect }\end{array}$ & $\begin{array}{l}\text { write } \\
\text { articl } \\
\text { research } \\
\text { effect } \\
\text { drug } \\
\text { result }\end{array}$ & $\begin{array}{l}\text { write } \\
\text { pitt } \\
\text { gordon } \\
\text { bank } \\
\text { articl } \\
\text { geb }\end{array}$ & $\begin{array}{l}\text { gordon } \\
\text { pitt } \\
\text { bank } \\
\text { geb } \\
\text { write } \\
\text { articl }\end{array}$ \\
\hline $\begin{array}{l}\text { card } \\
\text { board } \\
\text { comput } \\
\text { drive } \\
\text { controll } \\
\text { port }\end{array}$ & $\begin{array}{l}\text { comput } \\
\text { mac } \\
\text { card } \\
\text { drive } \\
\text { board } \\
\text { pc }\end{array}$ & $\begin{array}{l}\text { mac } \\
\text { comput } \\
\text { pc } \\
\text { hardwar } \\
\text { drive } \\
\text { appl }\end{array}$ & $\begin{array}{l}\text { mac } \\
\text { monitor } \\
\text { appl } \\
\text { comput } \\
\text { upgrad } \\
\text { hardwar }\end{array}$ & $\begin{array}{l}\text { monitor } \\
\text { mac } \\
\text { upgrad } \\
\text { research } \\
\text { appl } \\
\text { comput }\end{array}$ & $\begin{array}{l}\text { research } \\
\text { monitor } \\
\text { effect } \\
\text { upgrad } \\
\text { write } \\
\text { result }\end{array}$ & $\begin{array}{l}\text { research } \\
\text { effect } \\
\text { write } \\
\text { drug } \\
\text { result } \\
\text { articl }\end{array}$ & $\begin{array}{l}\text { write } \\
\text { effect } \\
\text { articl } \\
\text { research } \\
\text { result } \\
\text { year }\end{array}$ & $\begin{array}{l}\text { write } \\
\text { articl } \\
\text { effect } \\
\text { doctor } \\
\text { pitt } \\
\text { year }\end{array}$ & $\begin{array}{l}\text { pitt } \\
\text { write } \\
\text { articl } \\
\text { gordon } \\
\text { bank } \\
\text { geb }\end{array}$ \\
\hline $\begin{array}{l}\text { drive } \\
\text { controll } \\
\text { card } \\
\text { comput } \\
\text { disk } \\
\text { jumper }\end{array}$ & $\begin{array}{l}\text { drive } \\
\text { comput } \\
\text { pc } \\
\text { disk } \\
\text { mac } \\
\text { cabl }\end{array}$ & $\begin{array}{l}\text { mac } \\
\text { drive } \\
\text { comput } \\
\text { pc } \\
\text { port } \\
\text { hardwar }\end{array}$ & $\begin{array}{l}\text { mac } \\
\text { comput } \\
\text { drive } \\
\text { price } \\
\text { appl } \\
\text { upgrad }\end{array}$ & $\begin{array}{l}\text { mac } \\
\text { upgrad } \\
\text { comput } \\
\text { appl } \\
\text { monitor } \\
\text { write }\end{array}$ & \begin{tabular}{l|} 
write \\
effect \\
upgrad \\
articl \\
research \\
monitor
\end{tabular} & $\begin{array}{l}\text { write } \\
\text { effect } \\
\text { articl } \\
\text { year } \\
\text { research } \\
\text { result }\end{array}$ & $\begin{array}{l}\text { write } \\
\text { effect } \\
\text { doctor } \\
\text { articl } \\
\text { year } \\
\text { treatment }\end{array}$ & $\begin{array}{l}\text { write } \\
\text { doctor } \\
\text { effect } \\
\text { pain } \\
\text { articl } \\
\text { year }\end{array}$ & $\begin{array}{l}\text { pain } \\
\text { medic } \\
\text { write } \\
\text { doctor } \\
\text { articl } \\
\text { treat }\end{array}$ \\
\hline $\begin{array}{l}\text { drive } \\
\text { controll } \\
\text { disk } \\
\text { set } \\
\text { id } \\
\text { scsi }\end{array}$ & $\begin{array}{l}\text { drive } \\
\text { disk } \\
\text { comput } \\
\text { pc } \\
\text { set } \\
\text { cabl }\end{array}$ & $\begin{array}{l}\text { drive } \\
\text { mac } \\
\text { comput } \\
\text { disk } \\
\text { pc } \\
\text { write }\end{array}$ & $\begin{array}{l}\text { mac } \\
\text { drive } \\
\text { comput } \\
\text { price } \\
\text { write } \\
\text { articl }\end{array}$ & $\begin{array}{l}\text { mac } \\
\text { write } \\
\text { comput } \\
\text { articl } \\
\text { price } \\
\text { appl }\end{array}$ & $\begin{array}{l}\text { write } \\
\text { articl } \\
\text { comput } \\
\text { mac } \\
\text { year } \\
\text { upgrad }\end{array}$ & $\begin{array}{l}\text { write } \\
\text { articl } \\
\text { effect } \\
\text { year } \\
\text { school } \\
\text { comput }\end{array}$ & $\begin{array}{l}\text { write } \\
\text { doctor } \\
\text { articl } \\
\text { effect } \\
\text { year } \\
\text { treatment }\end{array}$ & $\begin{array}{l}\text { doctor } \\
\text { write } \\
\text { pain } \\
\text { articl } \\
\text { diseas } \\
\text { treatment }\end{array}$ & $\begin{array}{l}\text { pain } \\
\text { medic } \\
\text { doctor } \\
\text { write } \\
\text { treat } \\
\text { condition }\end{array}$ \\
\hline $\begin{array}{l}\text { drive } \\
\text { controll } \\
\text { disk } \\
\text { id } \\
\text { set } \\
\text { scsi }\end{array}$ & $\begin{array}{l}\text { drive } \\
\text { disk } \\
\text { pc } \\
\text { write } \\
\text { scsi } \\
\text { set }\end{array}$ & $\begin{array}{l}\text { drive } \\
\text { mac } \\
\text { disk } \\
\text { write } \\
\text { articl } \\
\text { pc }\end{array}$ & $\begin{array}{l}\operatorname{mac} \\
\text { write } \\
\text { drive } \\
\text { articl } \\
\text { price } \\
\text { comput }\end{array}$ & $\begin{array}{l}\text { write } \\
\text { articl } \\
\text { mac } \\
\text { appl } \\
\text { comput } \\
\text { price }\end{array}$ & $\begin{array}{l}\text { articl } \\
\text { comput } \\
\text { appl } \\
\text { school } \\
\text { port }\end{array}$ & $\begin{array}{l}\text { write } \\
\text { articl } \\
\text { school } \\
\text { year } \\
\text { water } \\
\text { comput }\end{array}$ & $\begin{array}{l}\text { write } \\
\text { articl } \\
\text { doctor } \\
\text { year } \\
\text { water } \\
\text { school }\end{array}$ & $\begin{array}{l}\text { write } \\
\text { doctor } \\
\text { articl } \\
\text { pain } \\
\text { treatment } \\
\text { medic }\end{array}$ & $\begin{array}{l}\text { medic } \\
\text { doctor } \\
\text { pain } \\
\text { write } \\
\text { treatment } \\
\text { diseas }\end{array}$ \\
\hline $\begin{array}{l}\text { drive } \\
\text { id } \\
\text { controll } \\
\text { disk } \\
\text { set } \\
\text { scsi }\end{array}$ & $\begin{array}{l}\text { drive } \\
\text { disk } \\
\text { write } \\
\text { articl } \\
\text { install } \\
\text { pc }\end{array}$ & $\begin{array}{l}\text { drive } \\
\text { write } \\
\text { articl } \\
\text { mac } \\
\text { disk } \\
\text { pc }\end{array}$ & $\begin{array}{l}\text { write } \\
\text { articl } \\
\text { mac } \\
\text { drive } \\
\text { price } \\
\text { appl }\end{array}$ & $\begin{array}{l}\text { write } \\
\text { articl } \\
\text { appl } \\
\text { mac } \\
\text { price } \\
\text { comput }\end{array}$ & $\begin{array}{l}\text { write } \\
\text { articl } \\
\text { appl } \\
\text { comput } \\
\text { school } \\
\text { machin }\end{array}$ & $\begin{array}{l}\text { write } \\
\text { articl } \\
\text { school } \\
\text { comput } \\
\text { year } \\
\text { water }\end{array}$ & $\begin{array}{l}\text { write } \\
\text { articl } \\
\text { doctor } \\
\text { school } \\
\text { year } \\
\text { person }\end{array}$ & $\begin{array}{l}\text { write } \\
\text { articl } \\
\text { doctor } \\
\text { treatment } \\
\text { peopl } \\
\text { medic }\end{array}$ & $\begin{array}{l}\text { medic } \\
\text { write } \\
\text { articl } \\
\text { treatment } \\
\text { doctor } \\
\text { pationt }\end{array}$ \\
\hline $\begin{array}{l}m b \\
\text { drive } \\
d x \\
b u \\
\text { controll } \\
\text { id }\end{array}$ & $\begin{array}{l}\text { write } \\
\text { articl } \\
\text { drive } \\
\text { mb } \\
\text { install } \\
\text { disk }\end{array}$ & $\begin{array}{l}\text { write } \\
\text { articl } \\
\text { mac } \\
\text { memori } \\
\text { install } \\
\text { drive }\end{array}$ & $\begin{array}{l}\text { write } \\
\text { articl } \\
\text { mac } \\
\text { appl } \\
\text { price } \\
\text { machin }\end{array}$ & $\begin{array}{l}\text { write } \\
\text { articl } \\
\text { appl } \\
\text { mac } \\
\text { price } \\
\text { comput }\end{array}$ & $\begin{array}{l}\text { articl } \\
\text { appl } \\
\text { comput } \\
\text { school } \\
\text { machin }\end{array}$ & $\begin{array}{l}\text { articl } \\
\text { school } \\
\text { comput } \\
\text { appl } \\
\text { peopl }\end{array}$ & $\begin{array}{l}\text { write } \\
\text { articl } \\
\text { peopl } \\
\text { person } \\
\text { doctor } \\
\text { year }\end{array}$ & $\begin{array}{l}\text { write } \\
\text { articl } \\
\text { peopl } \\
\text { doctor } \\
\text { person } \\
\text { year }\end{array}$ & $\begin{array}{l}\text { articl } \\
\text { write } \\
\text { medic } \\
\text { treatment } \\
\text { peopl } \\
\text { patient }\end{array}$ \\
\hline $\begin{array}{l}\text { dx } \\
\text { bu } \\
\text { mb } \\
\text { card } \\
\text { write } \\
\text { run }\end{array}$ & $\begin{array}{l}\text { write } \\
\text { mb } \\
\text { run } \\
\text { articl } \\
\text { memori } \\
\text { ram }\end{array}$ & $\begin{array}{l}\text { write } \\
\text { articl } \\
\text { mac } \\
\text { memori } \\
\text { machin } \\
\text { run }\end{array}$ & $\begin{array}{l}\text { write } \\
\text { appl } \\
\text { articl } \\
\text { mac } \\
\text { machin } \\
\text { price }\end{array}$ & $\begin{array}{l}\text { write } \\
\text { appl } \\
\text { articl } \\
\text { mac } \\
\text { comput } \\
\text { price }\end{array}$ & $\begin{array}{l}\text { articl } \\
\text { appl } \\
\text { comput } \\
\text { price } \\
\text { peopl }\end{array}$ & $\begin{array}{l}\text { write } \\
\text { articl } \\
\text { peopl } \\
\text { appl } \\
\text { comput } \\
\text { school }\end{array}$ & $\begin{array}{l}\text { articl } \\
\text { peopl } \\
\text { person } \\
\text { food } \\
\text { year }\end{array}$ & $\begin{array}{l}\text { articl } \\
\text { peopl } \\
\text { person } \\
\text { year } \\
\text { doctor }\end{array}$ & $\begin{array}{l}\text { articl } \\
\text { write } \\
\text { peopl } \\
\text { treatment } \\
\text { diseas } \\
\text { patient }\end{array}$ \\
\hline $\begin{array}{l}\text { bu } \\
\text { card } \\
\text { dx } \\
\text { mb } \\
\text { write } \\
\text { ram }\end{array}$ & $\begin{array}{l}\text { write } \\
\text { ram } \\
\text { card } \\
\text { run } \\
\text { mb } \\
\text { spe }\end{array}$ & $\begin{array}{l}\text { write } \\
\text { mac } \\
\text { articl } \\
\text { ram } \\
\text { run } \\
\text { memori }\end{array}$ & $\begin{array}{l}\text { appl } \\
\text { write } \\
\text { mac } \\
\text { articl } \\
\text { quadra } \\
\text { comput }\end{array}$ & $\begin{array}{l}\text { appl } \\
\text { write } \\
\text { articl } \\
\text { mac } \\
\text { comput } \\
\text { quadra }\end{array}$ & $\begin{array}{l}\text { appl } \\
\text { articl } \\
\text { comput } \\
\text { peopl } \\
\text { mac }\end{array}$ & $\begin{array}{l}\text { write } \\
\text { articl } \\
\text { peopl } \\
\text { appl } \\
\text { food } \\
\text { msg }\end{array}$ & $\begin{array}{l}\text { write } \\
\text { articl } \\
\text { peopl } \\
\text { food } \\
\text { msg } \\
\text { chines }\end{array}$ & $\begin{array}{l}\text { write } \\
\text { articl } \\
\text { peopl } \\
\text { food } \\
\text { year } \\
\text { msg }\end{array}$ & $\begin{array}{l}\text { peopl } \\
\text { write } \\
\text { diseas } \\
\text { treatment } \\
\text { studi }\end{array}$ \\
\hline
\end{tabular}

Fig. 5. The most probable words formed in each of the $10 \times 10$ grid points by the binomial latent trait model on two overlaping ("comp.sys.ibm.pc.harware," "comp.sys.mac.hardware,") and one distinct ("sci.med") classes of discussions from the 20 Newsgroups collection. Over a dictionary of 150 terms from each class, 200 documents were taken.

discussions we have specific subjects about video cards and monitors in the lower region, then moving up the grid, processor components, then internal memory, and finally, external memory in the upper subregion.

\subsection{Digits Data Compression and Visualization}

To see how the same algorithm can be applied to data coming from different representations in different contexts, an experiment on binary coded handwritten digits, described in the previous section is presented. There were 200 examples of each of the handwritten digits " 0, " " 1, " " 2, , " 3 ," and " 4 " formed the inputs of the Bernoulli trait algorithm. Fig. 7 shows three instances from each category. In this experiment, nine nonlinear and one linear basis vector were chosen to provide the samples from the assumed latent distribution. We perform a data compression from 240 dimensions to 2, going through the 10 basis functions. This setting implies broader neighborhood

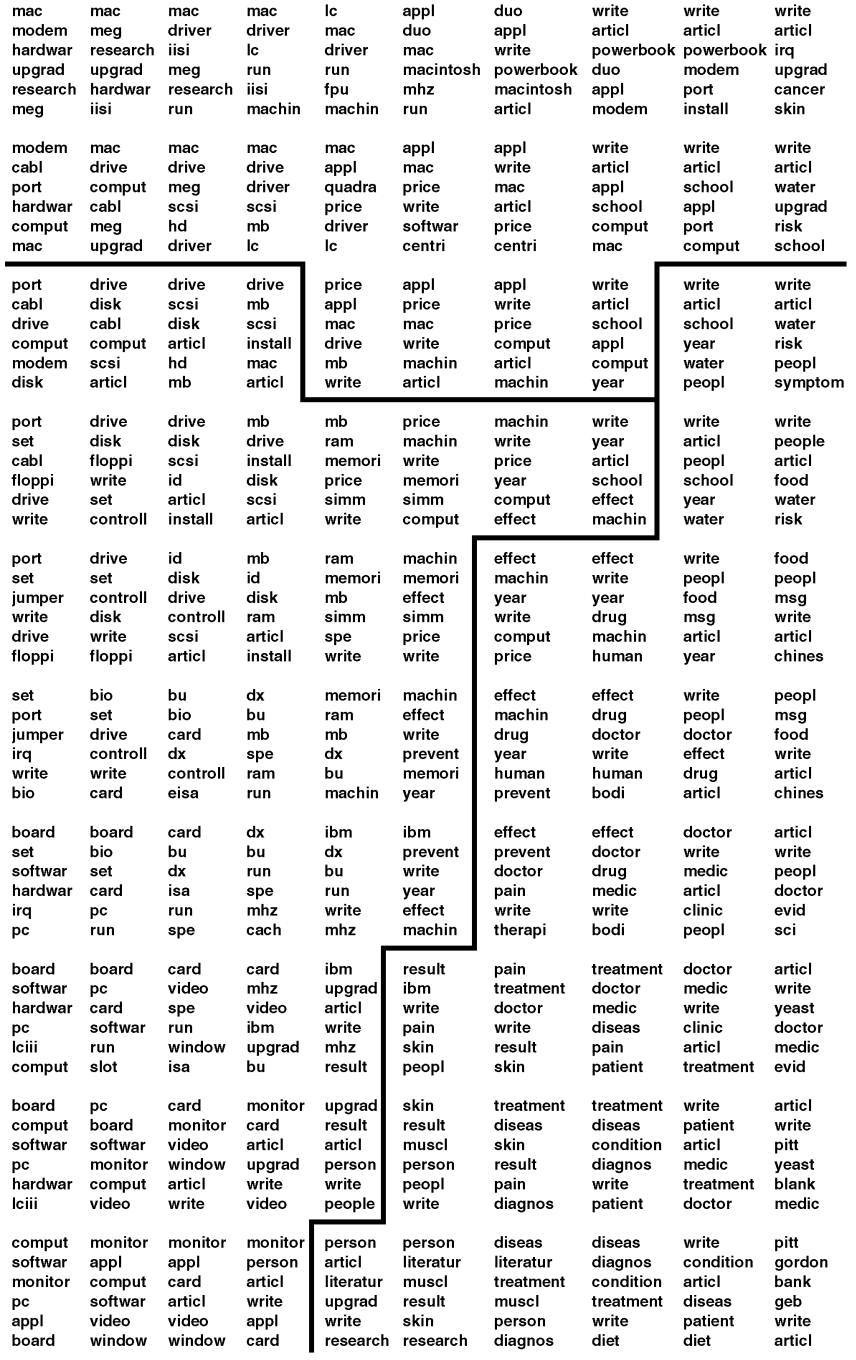

Fig. 6. The most probable words formed in each of the $10 \times 10$ grid points by the multinomial latent trait model on two overlapping ("comp.sys.ibm.pc.hardware," "comp.sys.mac.hardware,") and one distinct ("sci.med") classes of discussions from the 20 Newsgroups collection. Over a dictionary of 150 terms, 200 documents were taken from each class.

relations than the setting which was utilized for documents data and experimentally proved to be sufficient for this data-we found that different numbers of nonlinear basis functions provided qualitatively similar results.

Similar to the text-mining application, where the reference-vectors, i.e., the $\mathbf{g}\left(\mathbf{A} \mathbf{c}_{k}\right)$ values gave us the probability of each word for each of the $k=1 . . K$ grid-points, in the case of image-data, the same expression signifies the probabil-

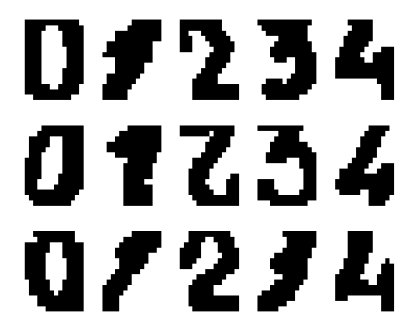

Fig. 7. The oberved data—three intstances from each class. 


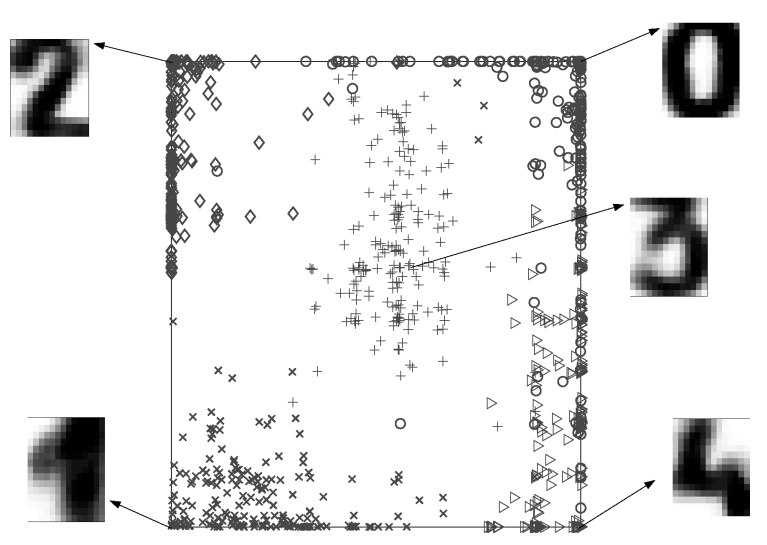

Fig. 8. Posterior means provided by the Bernoulli trait algorithm on a data set which consisted of handwritten digits and inspection of reference vectors in some of the grid points.

ities of each pixel in the reference-vector being active. In other words, we have a reference-image for each grid-point. The Bernoulli posterior means and the reference-images inspected in regions of agglomeration of the posterior mean projections are presented in Fig. 8.

For the binary coded digits data we found that the differences between the performance of an appropriate Bernoulli model and the Gaussian GTM model were much less pronounced. The reason is probably due to the more dense nature of this data than that of the document data.

More interesting is to see the representation that the algorithm has built and which underlies the topography of the $2 \mathrm{D}$ projection. The full set of reference-images corresponding to the $10 \times 10$ grid points are shown in Fig. 9. On this map, we can see not only the meaningful groupings of pixels, which indeed form images representing the four digits, but also a meaningful grouping of these referenceimages, as a macrostructure, in such a manner that neighboring images are geometrically more similar to each other than those found a further distance away. This is a basic feature of the presented topographic model. In addition, Fig. 9 illustrates the case where the trait algorithm works out intermediate forms, even if they are not represented by real examples, giving rise to a morphing between the different digits. These intermediate forms additionally create the blank areas between images of different classes on the 2D map of the posterior means and so help the partitioning in visualization.

\section{Conclusions}

We have presented a general probabilistic framework from which variants of class and trait models suitable for analysis, clustering and visualization of multivariate data follow. Models specifically applicable for (possibly sparse) discrete multivariate data were described. We also presented applications of the proposed models for text mining and digital image analysis. A readily interpretable representational structure was obtained on text based document data and an apparent topographic prototypical representation resulted in the case of images of handwritten digits.

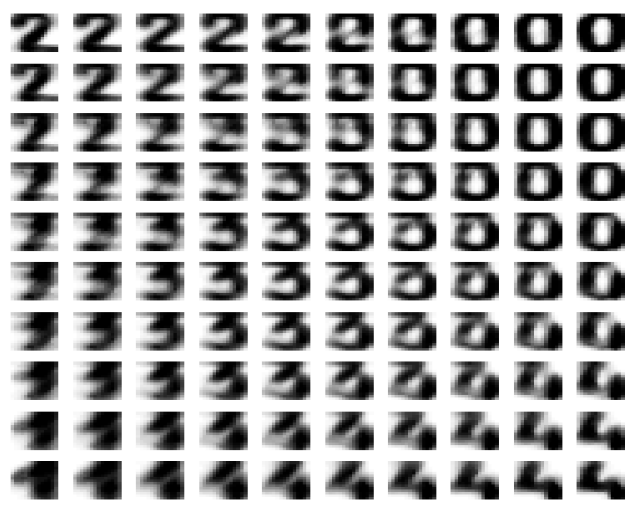

Fig. 9. The map of reference-images (i.e., the reshaped $\mathbf{g}\left(\mathbf{A c _ { k } )}\right.$ values, where $\mathbf{g}($.$) is component-wise sigmoid function in this case and$ $k=1 . .100$ ) formed on the first five handwritten digits classes. Neighboring grid points have similar reference-images. The morphing between the representation of the digits images is most apparent.

The presented method is useful for any such data analysis applications where the visualization of a multivariate discrete data set is sought.

\section{ApPEndix A}

\section{A.1 The Independent Bernoulli Noise Model}

Consider binary represented data. Each data point is a binary vector where the presence of a component is denoted by 1 and absence by 0 . In such cases, it is natural that the data vector be considered as being the statistical event, with the commonly used simplifying assumption that the underlying latent generative factors should capture all the dependency which exists between the components of the observed data i.e., the model noise should factorize.

$$
p\left(\mathbf{d}_{n} \mid \mathbf{c}=\mathbf{c}_{k}\right)=\prod_{t=1}^{T} p\left(d_{t n} \mid \mathbf{c}=\mathbf{c}_{k}\right) .
$$

Having to deal with binary data, the iid. Bernoulli noise is the usual assumption. The binomial Bernoulli distribution is one of the discrete members of the exponential family, namely specifying the cumulant function as

$$
G\left(\mathbf{A} \mathbf{c}_{k}\right)=\log \left\{1+\exp \left(\mathbf{A} \mathbf{c}_{k}\right)\right\} .
$$

This also specifies the link function, as the gradient of $G$ with respect to the natural parameter of the distribution:

$$
\mathbf{m}_{k}=g\left(\mathbf{A} \mathbf{c}_{k}\right)=\frac{\exp \left(\mathbf{A} \mathbf{c}_{k}\right)}{1+\exp \left(\mathbf{A} \mathbf{c}_{k}\right)}
$$

and the Fisher information matrix for the $k$ th latent unit is

$$
\mathbf{G}_{k}^{b i n}=\operatorname{diag}_{t}\left\{m_{t k}\left(1-m_{t k}\right)\right\},
$$

where $\operatorname{diag}(.)_{t}$ denotes a $T \times T$ diagonal matrix of its $t$-dependent argument as diagonal elements. The known form of the Bernoulli distribution and the generated divergence measure also emerges naturally from this: 


$$
\begin{aligned}
p\left(\mathbf{d}_{n} \mid \mathbf{c}_{k}\right) \propto \exp \left\{\mathbf{A} \mathbf{c}_{k} \mathbf{d}_{n}-\log \left(1+\exp \left(\mathbf{A} \mathbf{c}_{k}\right)\right)\right\} \\
=\exp \left\{\sum _ { t = 1 } ^ { T } \left\{d_{t n} \log \left(m_{t k}\right)\right.\right. \\
\left.\left.+\left(1-d_{t n}\right) \log \left(1-m_{t k}\right)\right\}\right\} \\
=\prod_{t=1}^{T} m_{t k}^{d_{t n}}\left(1-m_{t k}\right)^{1-d_{t n}}
\end{aligned}
$$

As can be seen from (25), the dissimilarity measure generated by the iid. Bernoulli noise assumption is the sum of component-wise cross entropies between the twovalued distributions generated by the data instance and reference vector, which can be rewritten as the sum of component-wise Kullback-Leibler divergences. Denoting by $\tilde{d}_{t n}$ and $\tilde{m}_{t k}$ the two valued probability distributions over the presence and absence of the $t$ th term in the $n$th datum and in the $k$ th prototype, respectively,

$$
\tilde{d}_{t n}\left\{\begin{array}{cc}
\text { presence } & \text { absence } \\
d_{t n} & 1-d_{t n}
\end{array}\right\} \tilde{m}_{t k}\left\{\begin{array}{cc}
\text { presence } & \text { absence } \\
m_{t k} & 1-m_{t k}
\end{array}\right\}
$$

so, $p\left(\mathbf{d}_{n} \mid \mathbf{c}_{k}\right)$ rewrites as

$$
p\left(\mathbf{d}_{n} \mid \mathbf{c}_{k}\right) \propto \exp \left\{-\sum_{t=1}^{T} \operatorname{KL}\left(\tilde{d}_{t n} \| \tilde{m}_{t k}\right)\right\} .
$$

So, maximizing the Bernoulli likelihood in fact minimizes the component-wise KL distances between the distributions made up by the datum and the corresponding reference vector.

\section{A.2 The Independent Poisson Noise Model}

Another discrete member of the exponential family, usually utilized in count data modeling, is the Poisson distribution. Similarly, specifying $G\left(\mathbf{A} \mathbf{c}_{k}\right)=\exp \left(\mathbf{A} \mathbf{c}_{k}\right)$, means specifying the Poisson distribution. Consequently, the link function now is

$$
\mathbf{m}_{k}=g\left(\mathbf{A} \mathbf{c}_{k}\right)=\exp \left(\mathbf{A} \mathbf{c}_{k}\right)
$$

and

$$
\mathbf{G}_{k}^{P o i}=\operatorname{diag}\left(m_{t k}\right) \text {. }
$$

Replacing the cumulant function in (4), we have

$$
\begin{aligned}
p\left(\mathbf{d}_{n} \mid \mathbf{c}_{k}\right) & \propto \exp \left\{\sum_{t=1}^{T}\left\{d_{t n} \log \left(m_{t k}\right)-m_{t k}\right\}\right\} \\
& \propto \prod_{t=1}^{T} \frac{1}{d_{t n} !} m_{t k}^{d_{t n}} \exp \left(-m_{t k}\right),
\end{aligned}
$$

from which can be seen the iid. Poisson noise generates the unnormalized KL divergence i.e., multiplying with $\exp \left(H\left(\mathbf{d}_{n}\right)+\mathbf{d}_{n}\right)$, which is independent on $\mathbf{m}_{k}$ we can rewrite

$$
\begin{aligned}
p\left(\mathbf{d}_{n} \mid \mathbf{c}_{k}\right) & \propto \exp \left\{-\sum_{t=1}^{T}\left\{d_{t n} \log \frac{d_{t n}}{m_{t k}}-m_{t k}+d_{t n}\right\}\right\} \\
& \propto \exp \left\{-K L_{u}\left(\mathbf{d}_{n} \| \mathbf{m}_{k}\right)\right\}
\end{aligned}
$$

\section{A.3 The Multinomial Noise Model}

The multinomial is another discrete member of the exponential family, which is specified by

$$
G\left(\mathbf{A} \mathbf{c}_{k}\right)=\log \left\{\sum_{t=1}^{T} \exp \left(\mathbf{A}_{t} \mathbf{c}_{k}\right)\right\}
$$

where $\mathbf{A}_{t}$ is the $t$ th row of $\mathbf{A}$. Consequently the link function in this case is

$$
\mathbf{m}_{k}=g\left(\mathbf{A} \mathbf{c}_{k}\right)=\frac{\exp \left(\mathbf{A}_{t} \mathbf{c}_{k}\right)}{\sum_{t^{\prime}=1}^{T} \exp \left(\mathbf{A}_{t^{\prime}} \mathbf{c}_{k}\right)}
$$

and

$$
\left(g_{t t^{\prime}}\right)_{k}{ }^{m u l}=\frac{\partial m_{t}\left(\mathbf{A c}_{k}\right)}{\partial \mathbf{A}_{t^{\prime}} \mathbf{c}_{k}}=m_{t k} \delta_{t t^{\prime}}-m_{t k} m_{t^{\prime} k},
$$

where $\delta_{t t^{\prime}}$ is the Kronecker delta function, 1 when $t=t^{\prime}$ and 0 otherwise. Thus, the form of the distribution is

$$
\begin{aligned}
p\left(\mathbf{d}_{n} \mid \mathbf{c}_{k}\right) & \propto \exp \left\{\sum_{t} d_{t n} \log \left(m_{t k}\right)\right\} \\
& =\prod_{t} m_{t k}^{d_{t n}}
\end{aligned}
$$

and the generated divergence is the negative cross-entropy, as can be seen from (35), which is proportional to the Kullback-Leibler divergence (if we multiply (35) by $\left.\exp \left(H\left(\mathbf{d}_{n}\right)\right)\right)$. So,

$$
p\left(\mathbf{d}_{n} \mid \mathbf{c}_{k}\right) \propto \exp \left\{-K L\left(\mathbf{d}_{n} \| \mathbf{m}_{k}\right)\right\} .
$$

The multinomial model is generally used in cases when the variables are nominal, indicating one of a number $n$ of mutually exclusive classes, in which case these variables are coded by $n$-dimensional binary vectors and only that component has the value 1 which corresponds to the class the variable belongs to and all others are 0 (1-of- $n$ coding scheme). However, the generated distance being the KL distance between a data point-seen as a discrete distribution over it's components-and the expectation parameter-which is also a discrete distribution over the same set -indicates the usefulness of the multinomial model in all those cases when the distributional interpretation of the data points is possible. As such, the multinomial is an alternative choice also for modeling count data. The choice should be driven by prior knowledge on the data, for efficient fitting.

\section{ACKNOWLEDGMENTS}

This work is funded by re:source, The Council for Museums Archives, and Libraries "Improved online information access," grant number RE/092. Part of this work was performed while the authors were visiting the Helsinki University of Technology under the TEKES visiting professor program. Some of the routines of the MATLAB GTM Toolbox http://www.ncrg.aston.ac.uk/GTM were used in the software for the reported simulations. The RainBow toolkit http:/ /www.cs.cmu.edu/mccallum/bow / 
was used for preprocessing the raw text data. The authors are most grateful to the anonymous reviewers for their insightful and helpful comments.

\section{References}

[1] S. Amari, Differential Geometrical Methods in Statistics. Berlin: Springer Verlag, 1985.

[2] O. Barndorff-Nielsen, Information and Exponential Families in Statistical Theory. Chichester: Wiley, 1978.

[3] A. Belouchrani and J.F. Cardoso, "A Maximum Likelihood Source Separation for Discrete Sources," Proc. European Signal Processing Conf., vol. 2, pp. 768-771, 1994.

[4] C.M. Bishop, Neural Networks for Pattern Recognition, pp. 215-234, Oxford Univ. Press, 1995.

[5] C.M. Bishop, M. Svensen, and C.K.I. Williams, "Developments of the Generative Topographic Mapping," Neurocomputing, vol. 21, pp. 203-224, 1998

[6] C.M. Bishop, M. Svensen, and C.K.I. Williams, "GTM: The Generative Topographic Mapping," Neural Computation, vol. 10, no. 1, pp. 215-235, 1998.

[7] P. Cheeseman and J. Stutz, "Bayesian Classification (AutoClass): Theory and Results," Advances in Knowledge Discovery and Data Mining. U.M. Fayyad, G. Piatetsky-Shapiro, P. Smyth and R. Uthurusamy eds., AAAI Press/MIT Press, 1996.

[8] P. Cheeseman, J. Kelly, M. Self, J. Stutz, W. Taylor, and D. Freeman, "AutoClass: A Bayesian Classification System," Proc. Fifth Int'l Conf. Machine Learning. 1988.

[9] K.W. Church and W. Gale, "Poisson Mixtures," Natural Language Eng., vol. 1, no. 2, pp. 163-190, 1995.

[10] T.M. Cover and J.A. Thomas, Elements of Information Theory, pp. 25-26, John Wiley and Sons, 1991.

[11] A.P. Dempster, N.M. Laird, and D.B. Rubin, "Maximum Likelihood from Incomplete Data via the EM Algorithm," J. Royal Statistical Soc., B, vol. 39, no. 1, pp. 1-38, 1977.

[12] S. Dumais, J. Platt, D. Heckerman, and M. Sahami, "Inductive Learning Algorithms and Representations for Text Categorization," Proc. ACM-Conf. Information and Knowlege Management 98, pp. 148-155, 1998.

[13] A.P. Dunmur and D.M. Titterington, "Analysis of Latent Structure Models with Multidimensional Latent Variables," Statistics and Neural Networks: Recent Advances at the Interface. J.W. Kay and D.M. Titterington, eds., pp. 165-194, Oxford: Oxford Univ. Press, 1999.

[14] S.T. Roweis and Z. Ghahramani, "A Unifying Review of Linear Gaussian Models," Neural Computation, vol. 11, no. 2, pp. 305-345, 1999.

[15] M. Girolami, "A Generative Model for Sparse Discrete Binary Data with Non-Uniform Categorical Priors," Proc. European Symp. Artificial Neural Networks (ESANN'00), pp. 1-6, 2000.

[16] M. Girolami, "Document Representations Based on Generative Multivariate Bernoulli Latent Topic Models," Proc. BCS-Information Retrival Specialist Group 22nd Ann. Colloquium Information Retrieval Research, pp. 194-201, 2000.

[17] M. Girolami, A. Cichocki, and S.I. Amari, "A Common Neural Network Model for Exploratory Data Analysis and Independent Component Analysis," IEEE Trans. Neural Networks, vol 9, no. 6, pp. 1495-1501, 1998.

[18] M. Girolami, Self-Organising Neural Networks. Springer-Verlag, 1999.

[19] M. Girolami, Advances in Independent Component Analysis. Perspectives in Neural Computation. M. Girolami, ed. Springer Verlag, 2000.

[20] I.J. Good, The Estimation of Probabilities: An Essay on Modern Bayesian Methods. M.I.T. Press, 1965.

[21] A. Hyvärinen, "Fast and Robust Fixed-Point Algorithms for Independent Component Analysis," IEEE Trans. Neural Networks, vol. 10, no. 3, pp. 626-634, 1999.

[22] T. Hofmann, "Learning the Similarity of Documents," Proc. Advances in Neural Information Processing Systems. MIT Press, 2000.

[23] T. Hofmann, "A Probabilistic Approach for Mapping Large Document Collections," J. Intelligent Data Analysis, http:// www.cs.brown.edu/people/th/publications.html

[24] A. Kabán and M. Girolami, "Initialized and Guided EMClustering of Sparse Binary Data with Application to Text Based Documents," Proc. 15th Int'l Conf. Pattern Recognition, vol. 2, pp. $748-751,2000$.
[25] A. Kabán and M. Girolami, "Clustering of Text Documents by Skewness Maximization," Proc. Second Int'l Workshop Independent Component Analysis and Blind Signal Separation, pp. 435-440, 2000.

[26] T. Kohonen, Self-Organizing Maps. Heidelberg: Springer, 1995.

[27] R. Kohavi, B. Backer, and D. Sommerfield, "Improving Simple Bayes," Proc. European Conf. Machnine Learning, 1997.

[28] D.D. Lee and H.S. Seung, "Learning the Parts of Objects by Nonnegative Matrix Factorization," Nature, vol. 401, pp. 788-791, 1999.

[29] D.D. Lewis, "Naive (Bayes) at Forty: The Independence Assumption in Information Retrieval," European Conf. Machine Learning, pp. 4-15, 1998.

[30] D.D. Lewis, "Feature Selection and Feature Extraction for Text Categorization," Proc. Speech and Natural Language Workshop, Defense, Advanced Research Projects Agency, pp. 212-217, 1992.

[31] D.J.C. MacKay, "Density Networks and their Application to Protein Modelling," Maximum Entropy and Bayesian Methods, pp. 259-268, Kluwer, 1996.

[32] D.J.C. MacKay, "Bayesian Neural Networks and Density Networks," Nuclear Instruments and Methods in Physics Research, Section A, vol. 354, no. 1, pp. 73-80, 1995.

[33] A. McCallum and K. Nigam, "A Comparison of Event Models for Naive Bayes Text Classification," Proc. Am. Assoc. Artifical Intelligence/Int'l Conf. Machine Learning-98 Workshop Learning Text Categorization, Technical Report WS-98-05, pp. 41-48, 1998.

[34] P. McCullagh and L.A. Nelder, Generalized Linear Models. Chapman and Hall, 1985.

[35] M. Meila and D. Heckerman, An Experimental Comparison of Several Clustering and Initialization Methods, Technical Report MSR-TR-98-06, Microsoft Research, Feb. 1998.

[36] F. Mosteller and D. Wallace, Applied Bayesian and Classical Inference. Springer-Verlag, 1984.

[37] K. Nigam, K. McCallum, S. Thrun, and T. Mitchell, "Text Classification from Labeled and Unlabeled Documents Using EM," Machine Learning, pp. 1-34, 1999.

[38] G.T. McLachlan and T. Krishnan, The EM Algorithm and Extensions. Wiley, 1997.

[39] I. Moustaki, "A Latent Trait and a Latent Class Model for Mixed Observed Variables," British J. Math. and Statistical Psychology, vol. 49, pp. 313-334, 1996.

[40] P. Pajunen, "Blind Separation of Binary Sources with Less Sensors than Sources," Proc. IEEE Int'l Conf. Neural Networks, vol. 3, pp. 1994-1997, 1997.

[41] M.A. Peot, "Geometric Implications of the Naïve Bayes Assumption," Proc. Conf. Uncertainty in Artificial Intelligence (UAI'96), pp. 414-419, 1996.

[42] M. Sahami, "Using Machine Learning to Improve Information Access," PhD thesis, Stanford Univ. 1998.

[43] M.D. Sammel, L.M. Ryan, and J.M. Legler, "Latent Variable Models for Mixed Discrete and Continuous Outcomes," J. Royal Statistical Soc., Series B, vol. 59, pp. 667-678, 1997.

[44] M.E. Tipping and C.M. Bishop, "Probabilistic Principal Component Analysis," J. Royal Statistical Soc., Series B, vol. 61, no. 3, pp. 611-622, 1999.

[45] M.E. Tipping, "Probabilistic Visualization of High-Dimensional Binary Data," Proc. Advances in Neural Information Processing Systems (NIPS*11), pp. 592-598, 1999.

[46] S. Vaithyanathan and B. Dom, "Generalized Model Selection for Unsupervised Learning in High Dimensions," Proc. Advances in Neural Information Processing Systems (NIPS*99), 1999.

[47] A. Vinokourov and M. Girolami, "A Probabilistic Hierarchical Clustering Method for Organizing Collections of Text Documents," Proc. Fifth Int'l Conf. Pattern Recognition (ICPR'2000), vol. 2, pp. 182-185, 2000.

[48] H.H. Yang and J. Moody, "Data Visualization and Feature Selection: New Algorithms for Nongaussian Data," Proc. Advances in Neural Information Processing Systems, pp. 687-693, MIT Press, 2000,

[49] Y. Yang and X. Liu, "A Re-Examination of Text Categorization Methods," Proc. ACM SIGIR Conf. Research and Development in Information Retrieval (SIGIR), pp. 42-49, 1999. 


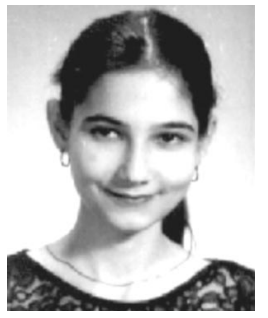

Ata Kabán received the BA degree with honors in musical composition, the $\mathrm{MA}$ and $\mathrm{PhD}$ degrees in musicology from the Music Academy "Gh. Dima" of Cluj-Napoca, Romania. Her thesis treated algebraic elements in 20th century's musical creation, defended against Professor Gh. Firca from Bucharest. She received the BSc degree with honors in computer science from "Babes-Bolyai" University of Cluj-Napoca, Romania. She is a research assistant at the University of Paisley, working on the development of probabilistic models for text-based information retrieval and from June to December 2000 was a visiting researcher at the Laboratory of Computer and Information Science, Helsinki University of Technology.

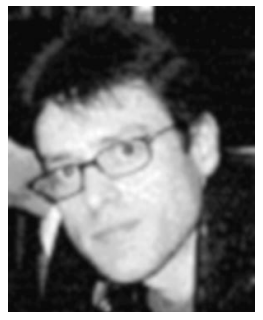

Mark Girolami received a degree in mechanical engineering from the University of Glasgow in 1985, and the PhD degree in computing science from the University of Paisley, 1998. He has recently been appointed to the chair of applied computational intelligence at the University of Paisley. From May to December 2000, he was the TEKES visiting professor at the Laboratory of Computer and Information Science in Helsinki University of Technology. In 1997 (June-August) and 1998 (May-October), he was a research fellow at the Laboratory for Advanced Brain Signal Processing at the Brain Science Institute, RIKEN, Wako-Shi, Japan. He has authored one book, edited one other, and has published more than 50 refereed publications in the general area of unsupervised learning. He was a development engineer with IBM from 1985 to 1995 when he left to pursue an academic career. He is a member of the IEEE.

$\triangleright$ For further information on this or any computing topic, please visit our Digital Library at http://computer.org/publications/dlib. 